\title{
Risk of Malignancy in Thyroid Nodules Using the American College of Radiology Thyroid Imaging Reporting and Data System in the NIFTP Era
}

Authors

Pedro Weslley Rosario, Alexandre Lemos da Silva, Maurício Buzelin Nunes, Michelle Aparecida Ribeiro Borges

\author{
Affiliation \\ Santa Casa de Belo Horizonte, Minas Gerais, Brazil \\ Key words \\ thyroid nodule, ACR TI-RADS, ultrasonography, \\ risk of malignancy, NIFTP \\ received $\quad 10.05 .2018$ \\ accepted 11.09.2018 \\ Bibliography \\ DOI https://doi.org/10.1055/a-0743-7326 \\ Horm Metab Res 2018; 50: 735-737 \\ (c) Georg Thieme Verlag KG Stuttgart · New York \\ ISSN 0018-5043 \\ Correspondence \\ P. W. Rosario, MD \\ Instituto de Ensino e Pesquisa da Santa Casa de Belo \\ Horizonte \\ Rua Domingos Vieira, 590 \\ Santa Efigênia CEP 30150-240 \\ Belo Horizonte MG \\ Brazil \\ Tel.: + 55/31/32388 819, Fax: + 55/31/32388980 \\ pedrowsrosario@gmail.com
}

\begin{abstract}
Recently, the American College of Radiology (ACR) proposed a Thyroid Imaging Reporting and Data System (TI-RADS) for thyroid nodules based on ultrasonographic features. It is important to validate this classification in different centres. The present study evaluated the risk of malignancy in solid nodules $>1 \mathrm{~cm}$ using ACR TI-RADS. The risk of malignancy was defined including noninvasive follicular thyroid neoplasm with papillary-like nuclear features (NIFTP) and after its exclusion from malignant tumours. For the present study, the original images were revised, and each nodule was assigned to one of the TI-RADS levels proposed for solid nodules: TR3, TR4, or TR5. This risk of malignancy was significantly different for the three levels: $1.7 \%, 11.2 \%$, and $60.6 \%$ for TR3, TR4, and TR5, respectively, when NIFTP was included, and $0.6 \%, 7.9 \%$, and $60.2 \%$ for TR3, TR4, and TR5, respectively, when NIFTP was excluded from malignant tumours. The nodules corresponding to NIFTP were classified according to ACR as TR3 in $28.5 \%$ of cases, TR4 in $67.8 \%$, and TR5 in only $3.5 \%$. The nodules corresponding to cancer were classified according to ACR as TR3 in only $2.3 \%$ of cases, TR4 in $27 \%$, and TR5 in $70.5 \%$. In conclusion, this study shows the validity of the ACR TI-RADS for solid thyroid nodules, even after the exclusion of NIFTP from malignant tumours.
\end{abstract}

\section{Introduction}

Recently, the American College of Radiology (ACR) proposed a Thyroid Imaging Reporting and Data System (TI-RADS) for thyroid nodules based on ultrasonographic features that consists of five levels [1]. Different from the classification of the American Thyroid Association (ATA) [2] in which some nodules may not meet the criteria for any of the categories [3-5], the TI-RADS permits to classify all nodules. However, it is important to validate this classification in different centres. Diagnostic fine-needle aspiration (FNA) is not recommended for TR1 (benign) and TR2 (not suspicious) nodules [1]. Thus, greater interest exists in the risk of malignancy of TR3 (mildly suspicious), TR4 (moderately suspicious), and TR5 (highly suspicious) nodules.

In the 4th edition of the World Health Organization classification of tumours of endocrine organs, the noninvasive encapsulat- ed follicular variant of papillary thyroid carcinoma (EFVPTC) is no longer considered "cancer" [6]. These tumours are now called "noninvasive follicular thyroid neoplasm with papillary-like nuclear features" (NIFTP) [6]. However, few studies have re-evaluated the risk of malignancy according to the ultrasonographic appearance of the nodule after the exclusion of NIFTP $[7,8]$.

We previously evaluated the risk of malignancy in thyroid nodules $>1 \mathrm{~cm}$ using the ultrasonographic classification of ATA [3,7]. The risk of malignancy was defined including NIFTP [3] and after its exclusion from malignant tumours [7]. The present study evaluated the risk of malignancy of these nodules using the ACR TI-RADS [1].

\section{Patients and Methods}

This study is derived from a prospective study $[3,7]$ that evaluated the ultrasonographic features of 1502 nodules $>1 \mathrm{~cm}$. These nod- 
ules were solid or predominantly solid and nontoxic in 1106 patients, all of them submitted to ultrasonography (US)-guided FNA and the results of cytology classified using the Bethesda system [2]. As reported previously [3, 7], there were 1005 nodules with benign cytology (on two occasions in the case of nodules with highly suspicious US findings) and 497 with nonbenign cytology, with histology being obtained from $485 / 497$ (97.6\%).

For the present study, the original images were revised separately by two professionals experienced in thyroid imaging and each nodule was assigned to one of the TI-RADS levels proposed for solid nodules [1]: TR3, TR4, or TR5. Despite disagreement on the sum of points in 20 nodules (difference of only 1 point), there was agreement between the two examiners on TI-RADS level in all nodules.

Fisher's exact test or the $x^{2}$ test was used to detect differences in the proportion of cases. A p-value $<0.05$ was considered to be significant.

\section{Results}

The risk of malignancy of each TI-RADS level is reported in > Table 1. This risk was significantly different for the three levels to which solid nodules can be assigned: $1.7 \%, 11.2 \%$, and $60.6 \%$ for TR3, TR4, and TR5 ( $p<0.0001)$, respectively, when NIFTP was included, and $0.6 \%$, $7.9 \%$, and $60.2 \%$ for TR3, TR4, and TR5 ( $p<0.001$ ), respectively, when NIFTP was excluded from malignant tumours.

The nodules corresponding to NIFTP $(n=28)$ were classified according to ACR as TR3 in $28.5 \%$ of cases, TR4 in $67.8 \%$, and TR5 in only $3.5 \%$. Much differently, the nodules corresponding to cancer ( $n=170$ ) were classified according to ACR as TR3 in only $2.3 \%$ of cases, TR4 in $27 \%$, and TR5 in $70.5 \%$.

\section{Discussion}

Our results favour the use of the ACR TI-RADS classification [1]. First, in contrast to the ATA classification [2] in which $4 \%$ of the nodules did not meet the criteria for any of the categories and had a risk of malignancy of $16 \%$ [3], in the present series all nodules could be classified using the ACR TI-RADS. In two other studies, 5\% [5] and 14\% [4] of the nodules were not defined using the ATA classification, with

- Table 1 Risk of malignancy in solid thyroid nodules $>1 \mathrm{~cm}$ according to the TI-RADS of the American College of Radiology [1].

\begin{tabular}{|l|c|c|}
\hline $\begin{array}{l}\text { TI-RADS [1] (number } \\
\text { of nodules) }\end{array}$ & \multicolumn{2}{|c|}{$\begin{array}{c}\text { Risk of malignancy } \\
\text { interval) }\end{array}$} \\
& Malignant NIFTP & confidence \\
& Non-malignant NIFTP \\
\hline TI-RADS 3 $(n=694)$ & $1.7 \%(0.96-3 \%)$ & $0.6 \%(0.17-1.5 \%)$ \\
\hline TI-RADS $4(n=580)$ & $11.2 \%(8.9-14 \%)$ & $7.9 \%(6-10.4 \%)$ \\
\hline TI-RADS 5 $(n=216)$ & $56 \%(49.3-62.5 \%)$ & $55.5 \%(49-62 \%)$ \\
\hline
\end{tabular}

TI-RADS: Thyroid Imaging Reporting and Data System; NIFTP: Noninvasive Follicular Thyroid Neoplasm with Papillary-like nuclear features. malignancy in 15\% [5] and 10\% [4], respectively. Second, since the sum of points ranges from 4 to 6 for level TR3 and is 7 or higher for level TR5, the small difference (1 point) in the sum of points observed for few nodules did not change the TI-RADS level, with $100 \%$ agreement between the two examiners. This excellent agreement was obtained with only two examiners who work in the same research centre and have large experience with thyroid US. It should be noted that significant disagreement may be observed when many professionals with variable experience who work in different places (research institutions or private companies) are involved [9]. Third, a large difference in the risk of malignancy was observed between the three TI-RADS levels to which solid nodules can be assigned [1]. The finding that calls attention is the very low risk of malignancy observed for TR3 nodules ( $\leq 3 \%$ ) and, conversely, the high risk found for TR5 nodules ( $\geq 50 \%$ ). Some comparative studies suggested superior performance of ACR TI-RADS over the ATA classification [4, 5].

An important issue addressed in this study was the impact of noninvasive EFVPTC on the risk of malignancy. Until now, in the series that evaluated the risk of malignancy according to the ultrasonographic features of the nodules, these tumours were considered malignant $[4,5,10]$, including the multicentre study validating the ACR TI-RADS in the United States [10]. With the recent change that no longer considers these tumours to be malignant [6], the originally reported malignancy rates might be lower, at least for some TI-RADS levels, since the distribution of NIFTP is not uniform at the different levels. When we recalculated the risk of malignancy after excluding NIFTP, this rate was $0.6 \%$ for TR3 nodules and $8 \%$ for TR4 nodules, while no change was observed for TR5 nodules. Thus, a reduction in the risk of malignancy is possible in the case of TR3 and TR4 nodules but does not appear to occur in TR5 nodules. Only two studies have evaluated the impact of excluding NIFTP on the risk of malignancy estimated by US but did not use ACR TI-RADS. In the first study using the ATA classification, we showed a reduction in the risk of malignancy for low- and intermediate-suspicion nodules [7]. In the second study, Chaigneau et al. [8], using the French TIRADS for nodules with indeterminate cytology, demonstrated a reduction in the risk of malignancy mainly for TIRADS 4 A nodules. Like in the present study, in these two, the risk of malignancy in more suspicious nodules (high suspicion by ATA [7] and French TIRADS 4B or 5 [8]) did not change after the exclusion of NIFTP.

Our study is also the first to evaluate ACR TI-RADS in NIFTP. We showed that TR4 was the most frequent, TR5 was uncommon (3.5\%), and TR3 was common in this neoplasm, in contrast to carcinomas in which TR5 corresponded to $70 \%$ of cases and TR3 was rare $(<3 \%)$.

In conclusion, this study confirms the validity of the ACR TI-RADS [1] for solid thyroid nodules, even after the exclusion of NIFTP from malignant tumours [4], and shows the ultrasonographic appearance of this neoplasm using ACR TI-RADS.

\section{Compliance with Ethical Standards}

The study was approved by the Research Ethics Committee of our institution. Informed consent was obtained from all individual participants included in the study. 


\section{Funding}

This work was supported by the Brazilian National Council for Scientific and Technological Development (CNPq).

\section{Conflict of Interest}

The authors declare that they have no conflict of interest.

\section{References}

[1] Tessler FN, Middleton WD, Grant EG, Hoang JK, Berland LL, Teefey SA, Cronan J], Beland MD, Desser TS, Frates MC, Hammers LW, Hamper UM, Langer JE, Reading CC, Scoutt LM, Stavros AT. ACR Thyroid Imaging, Reporting and Data System (TI-RADS): White Paper of the ACR TI-RADS Committee. J Am Coll Radiol 2017; 14: 587-595

[2] Haugen BR, Alexander EK, Bible KC, Doherty GM, Mandel S], Nikiforov YE, Pacini F, Randolph GW, Sawka AM, Schlumberger M, Schuff KG, Sherman SI, Sosa JA, Steward DL, Tuttle RM, Wartofsky L. 2015 American Thyroid Association Management Guidelines for Adult Patients with Thyroid Nodules and Differentiated Thyroid Cancer: The American Thyroid Association Guidelines Task Force on Thyroid Nodules and Differentiated Thyroid Cancer. Thyroid 2016; 26: 1-133

[3] Rosario PW, da Silva AL, Nunes MS, Ribeiro Borges MA, Mourão GF, Calsolari MR. Risk of malignancy in 1502 solid thyroid nodules $>1 \mathrm{~cm}$ using the new ultrasonographic classification of the American Thyroid Association. Endocrine 2017; 56: 442-445
[4] Middleton WD, Teefey SA, Reading CC, Langer JE, Beland MD, Szabunio MM, Desser TS. Comparison of Performance Characteristics of American College of Radiology TI-RADS, Korean Society of Thyroid Radiology TIRADS, and American Thyroid Association Guidelines. AJR Am J Roentgenol 2018; 210: 1148-1154

[5] Lauria Pantano A, Maddaloni E, Briganti SI, Beretta Anguissola G, Perrella E, Taffon C, Palermo A, Pozzilli P, Manfrini S, Crescenzi A. Differences between ATA, AACE/ACE/AME and ACR TI-RADS ultrasound classifications performance in identifying cytological high-risk thyroid nodules. Eur J Endocrinol 2018; 178: 595-603

[6] Lloyd RV, Osamura RY, Klöppel G, Rosai J. WHO Classification of Tumours of Endocrine Organs. 4th edition, Lyon: IARC; 2017

[7] Rosario PW, Silva TH, de Oliveira PHL. Impact of noninvasive follicular thyroid neoplasm with papillary-like nuclear features (NIFTP) on the risk of malignancy estimated by the ultrasonographic classification of the American Thyroid Association (ATA) in thyroid nodules $>1 \mathrm{~cm}$. Endocrine 2018; 60: 535-536

[8] Chaigneau E, Russ G, Royer B, Bigorgne C, Bienvenu M, Rouxel A, Leenhardt L, Belin L, Buffet C. TIRADS score is of limited clinical value for risk stratification of indeterminate cytological results. Eur J Endocrinol 2018; 179: 13-20

[9] Hoang JK, Middleton WD, Farjat AE, Teefey SA, Abinanti N, Boschini F], Bronner AJ, Dahiya N, Hertzberg BS, Newman JR, Scanga D, Vogler RC, Tessler FN. Interobserver Variability of Sonographic Features Used in the American College of Radiology Thyroid Imaging Reporting and Data System. AJR Am J Roentgenol 2018; 211: 162-167

[10] Middleton WD, Teefey SA, Reading CC, Langer JE, Beland MD, Szabunio MM, Desser TS. Multiinstitutional Analysis of Thyroid Nodule Risk Stratification Using the American College of Radiology Thyroid Imaging Reporting and Data System. AJR Am J Roentgenol 2017; 208: 1331-1341 